\title{
Design and Initial Result of Time-Resolved Vertical Soft X-Ray Imaging System in Low- $A$ RFP*)
}

\author{
Akio SANPEI, Kensuke OKI, Daisuke FUKAHORI, Kazuaki DEGUCHI, Seiya NAKAKI, \\ Kanae NISHIMURA, Haruhiko HIMURA, Sadao MASAMUNE, Satoshi OHDACHI ${ }^{1)}$, \\ Nobuhiro NISHINO ${ }^{2)}$ and Takumi $\mathrm{ONCHI}^{3)}$ \\ Kyoto Institute of Technology, Kyoto 606-8585, Japan \\ ${ }^{1)}$ National Institute for Fusion Science, Toki 509-5292, Japan \\ ${ }^{2)}$ Hiroshima University, Hiroshima 739-8527, Japan \\ ${ }^{3)}$ University of Saskatchewan, Saskatchewan, Canada
}

(Received 9 December 2011 / Accepted 16 March 2012)

\begin{abstract}
A time-resolved vertical soft X-ray (SXR) imaging system was constructed. To identify the structure of the magnetic island using the obtained SXR images, we calculated the two-dimensional (2-D) image expected from low-aspect-ratio (low- $A$ ) reversed field pinch (RFP) plasmas. The 2-D image was found to reflect the change in the radiation power caused by the magnetic island. A preliminary experimental result in which we observed vertical SXR images from the quasi-periodic quasi-single helicity (QSH) state in low- $A$ RFP plasmas is presented. We successfully obtained the time evolution of SXR images from the vertical port. By comparing the observed images with simulated images, we found that the evolution of the experimental SXR image suggests a rotating helical configuration.
\end{abstract}

(C) 2012 The Japan Society of Plasma Science and Nuclear Fusion Research

Keywords: RFP, soft X-ray (SXR), imaging, magnetic island, high-speed camera

DOI: $10.1585 /$ pfr.7.2402052

\section{Introduction}

The measurement of bremsstrahlung soft X-ray (SXR) radiation is a useful passive method for diagnosing hightemperature plasmas because the contours of the SXR emissivity correspond to the magnetic surfaces of the plasmas. SXR imaging has been applied to high-temperature toroidal plasma experiments for the study of pressure fluctuations either in the core or at the periphery [1].

In the reversed field pinch (RFP), the toroidal pitch of the equilibrium field is relatively short, and the fluctuation component resulting from internally resonant global instabilities forms a three- dimensional (3-D) helical structure. Equilibrium analyses have shown that the innermost mode's rational surface can be located away from the axis in a lower aspect ratio $(A=R / a)$ RFP configuration, where $R(a)$ is the major (minor) radius of the plasma column. Therefore, a single mode can be expected to grow to a higher amplitude in a low- $A$ RFP than in medium- and high- $A$ RFPs. This may allow easier access to the quasisingle helicity (QSH) state, in which the internally resonant single tearing mode grows significantly larger than other modes. In the low- $A$ RFP machine RELAX $[2,3],(R$ $=0.51 \mathrm{~m}, a=0.25 \mathrm{~m}(A=2))$, a quasi-periodic transition to the QSH state has been observed [4]. During the QSH state, the fluctuation power is concentrated in the dominant

author'se-mail: sanpei@kit.ac.jp

*) This article is based on the presentation at the 21st International Toki Conference (ITC21). single $m=1$ mode.

We have developed SXR imaging diagnostics using multiple SXR cameras which consists a microchannel plate (MCP) and a fluorescent plate for identifying the structures of dominant magnetohydrodynamic (MHD) instabilities in the QSH state in the RFP [5,6]. The detailed design of the SXR camera has been reported elsewhere [7]. Moreover, we have constructed a fast successive SXR imaging system. As a preliminary experiment, we have taken tangential SXR pin-hole pictures with a time resolution of $10 \mu \mathrm{s}$ in order to identify the time evolution of a simple helix structure in RELAX plasmas [8,9]. As the next step, we have developed an SXR imaging diagnostic system that uses multiple pin-hole SXR cameras together with high-speed cameras to record the time evolution of the SXR images from the tangential and vertical directions simultaneously for studying the dynamic structures of 3-D SXR emissivity. Using this system, we expect to be able to discuss the 3-D dynamics of MHD instabilities associated with the QSH state.

In this paper, we describe the design and configuration of the vertical SXR imaging system. Quantitative analyses are explained in terms of both the phase and radial position of the magnetic island in the RFP. A preliminary experimental result obtained from the vertical port is presented. Then, the obtained experimental results and a comparison with simulated images are discussed. 


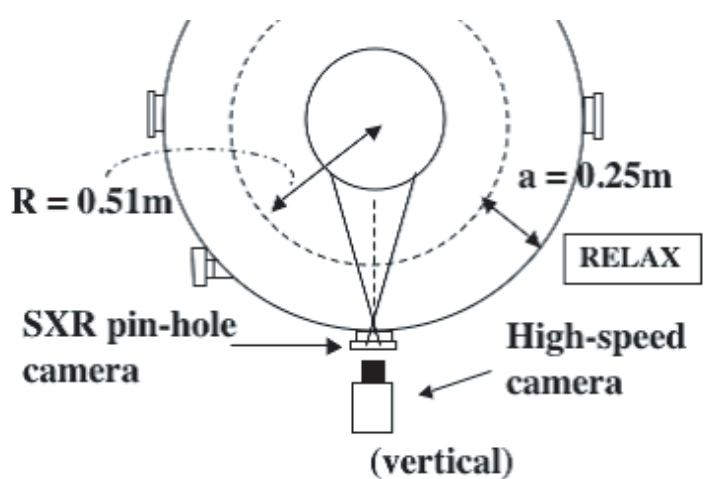

Fig. 1 Arrangement of the SXR pin-hole camera and the highspeed camera. Solid lines and dashed line represent the limits and the sight line of the center of the visual field, respectively.

\section{Design of SXR Imaging System and Calculation of 2-D Image for Verti- cal Observation}

A schematic drawing of the imaging system is shown in Fig. 1. The solid lines and a dashed line represent the limits and the sight line of the center of the visual field, respectively. This SXR imaging system uses an MCP to record a higher-resolution distribution of the twodimensional (2-D) luminosity on a phosphor plate. The energy efficiency of the MCP is nearly constant in the wavelength range of SXR from the RELAX plasma, and a linear relationship between the number of electrons ejected from the MCP and the luminosity on the phosphor plate has been confirmed $[10,11]$. The projected images depend strongly on the equilibrium magnetic field. It is difficult to clarifying the correlation between the filament structure associated with a high toroidal mode number $n$ and a projected tangential image. In this sense, the vertical imaging system mitigates the shortcomings of the tangential system.

In the vertical system, an indium tin oxide based phosphor plate is employed to cover the shortfall in the luminosity in images caused by the short sight line. The pinhole is placed at $172 \mathrm{~mm}$ horizontally (along the major radius) away from the plasma edge. The 2-D luminosity distributions corresponding to the integrated SXR emissivity are measured with a high-speed camera (Photoron FASTCAM SA-4) with an image size of $264 \times 96$ pixels array and a 12-bit dynamic range. Nevertheless, the SXR image is produced only in a limited area on the pixel array: $70 \times 70$ pixels.

To identify the magnetic islands of the RFP by referring to the SXR image, we first estimated the 2-D image on the phosphor plate $P_{\text {img }}$ expected from low- $A$ RFP plasmas. In our calculations, we assume the profiles of the radiation power from the magnetic island $P_{\text {rad-mi }}$ as follows. Considering a toroidal plasma, the electron density $n_{\mathrm{e}}(0)$, electron temperature $T_{\mathrm{e}}(0)$, and $P_{\text {rad-mi }}(0)$ on the plasma axis are assumed to be $1.00 \times 10^{19} / \mathrm{m}^{3}, 1.00 \times 10^{2} \mathrm{eV}$,

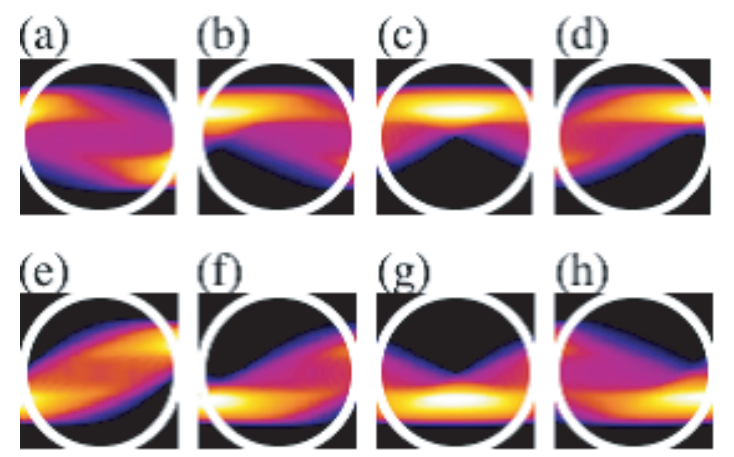

Fig. 2 Calculated 2-D luminosity distributions on phosphor plate due to the radiation power emitted from the $m=$ $1 / n=4$ magnetic island located at $r=6 \mathrm{~cm}$. The phase of the magnetic island changed by $0.25 \pi$ in each succeeding image.

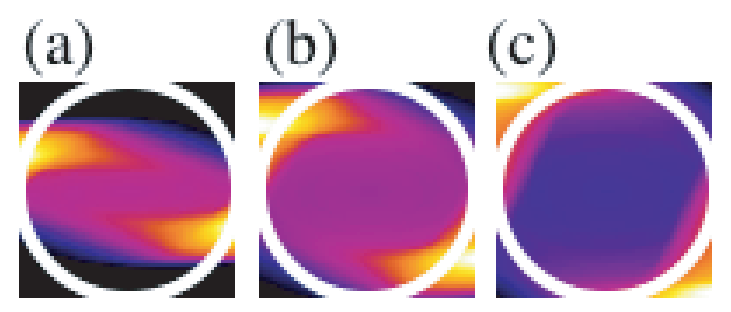

Fig. 3 Dependence of $P_{\text {img }}$ on the radial position of the magnetic island. The $m=1 / n=4$ magnetic island is located at $r=$ (a) $6 \mathrm{~cm}$, (b) $10 \mathrm{~cm}$ and (c) $14 \mathrm{~cm}$.

and $5.72 \times 10^{-17} \mathrm{~W} / \mathrm{cm}^{3}$, respectively. The power of the bremsstrahlung SXR radiation $P_{\text {rad }}$ is written as [12]

$$
P_{\text {rad }}=1.54 \times 10^{-32} n_{\mathrm{e}} n_{\mathrm{i}} Z^{2}\left(\kappa T_{\mathrm{e}}\right)^{1 / 2} \overline{g_{\mathrm{ff}}}\left(Z, T_{\mathrm{e}}\right),
$$

where $n_{\mathrm{i}}$ is the ion density, $Z$ is the effective ion charge, and $\overline{g_{\mathrm{ff}}}$ is the temperature-averaged free-free Gaunt factor. Here, $n_{\mathrm{e}}$ and $n_{\mathrm{i}}$ are assumed to be equal, and $Z$ is unity. Therefore, $P_{\text {rad }}(x, y, z)$ can be determined from $n(x, y, z)$ and $T_{\mathrm{e}}(x, y, z)$. Also, as eq. (1) shows, $P_{\text {rad }}$ is proportional to $n_{\mathrm{e}}^{2}$ and $T_{\mathrm{e}}^{1 / 2}$. Next, after calibrating the inherent sensitivity of the MCP to the incident angle of SXR, the value of $P_{\text {rad }}(x, y, z)$ is summed along the line of sight. Finally, the 2-D image of the SXR distribution $P_{\text {img }}(x, y)$ is obtained.

$P_{\text {img }}(x, y)$ has been calculated by assuming a single bean-shaped magnetic island due to a tearing mode at $m=1 / n=4$. Typical calculated 2-D images are shown in Figs. 2 and 3, which show the dependences of $P_{\text {img }}$ on $P_{\text {rad-mi }}(x, y, z)$ and the phase (Fig. 2) and the radial position of the magnetic island (Fig. 3), respectively. White circles indicate the field of vision. In Fig. 2, when the magnetic island due to the tearing mode is rotating, the calculated 2-D image reflecting its phase changes considerably. Figure 3 shows the results when the peak of $P_{\text {rad-mi }}$ is located at $r=$ (a) $6 \mathrm{~cm}$, (b) $10 \mathrm{~cm}$, and (c) $14 \mathrm{~cm}$. Comparing these results reveals that it becomes difficult to detect the peak in the luminosity as the radial position of $P_{\text {rad-mi }}$ increases. 


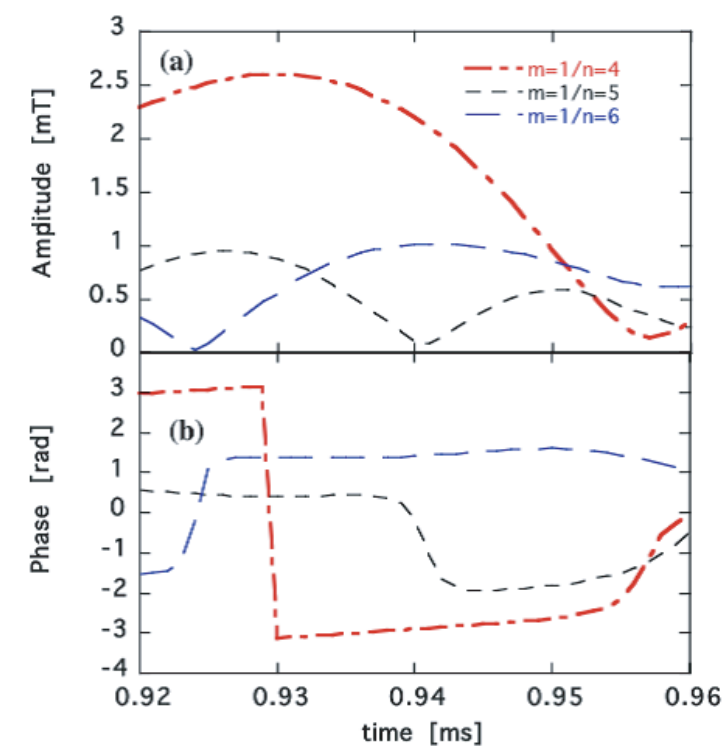

Fig. 4 Time evolution of the amplitude in $m=1 / n=4,5,6$ modes (a), the phase of the $m=1 / n=4,5,6$ modes (b).

\section{Experimental Result and Compari- son with Calculations}

In the low- $A$ RFP machine RELAX, a quasi-periodic transition to a QSH state in which the internally resonant single tearing mode grows significantly larger than other modes has been observed [4]. During the QSH state, the fluctuation power is concentrated in the single dominant $m$ $=1$ mode. Figure 4 (a) shows the time evolution of the amplitudes of the $m=1 / n=4,5,6$ modes obtained by edge measurement at an expanded time scale. In this time window, the dominant mode is the $m=1 / n=4$ mode until $950 \mu$ s. In Fig. 4 (b), we compare the time evolution of the phase of the $m=1 / n=4$ mode to that of the $m=1 / n=5,6$ modes. The phase of the $m=1 / n=4$ mode changes slowly while corresponding mode is dominant.

Raw images of SXR emission contain both equilibrium and fluctuating components. To illustrate the fluctuating component clearly, we applied a subtraction technique that enhances the difference between successive images, thus showing only the changes in the interval between images. In an acquisition of consecutive image frames $I_{\mathrm{n}}$, the $n$-th subtracted image in the sequence is given by $I_{\mathrm{n}}-I_{\mathrm{n}-1}$. Figure 5 shows subtracted images of the emissivity structure obtained from the vertical port in RELAX with a time resolution of $150 \mathrm{kfps}$. A zonal structure moves to the upper or left direction with time. In Fig. 5 (d), this zonal structure can be recognized even though the amplitude of the $m=1 / n=4$ mode is comparable with that of other modes, as shown in Fig. 4 (a). It is cited as a possible cause that the islands do not overlap because of this mode's small amplitudes. Moreover, the $m=1 / n=5$ and 6 modes may have lower emissivity because the corresponding rational surfaces are located outside that of $m=1 / n=4$. Figure 6 shows the time evolution of the vertical $(Z)$ luminosity pro-

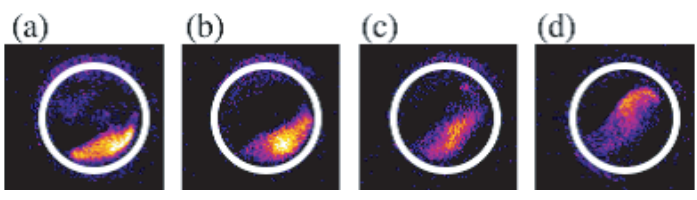

Fig. 5 Subtracted images of emissivity structure obtained from vertical port in RELAX at (a) $933 \mu \mathrm{s}$, (b) $940 \mu \mathrm{s}$, (c) $947 \mu \mathrm{s}$, and (d) $953 \mu \mathrm{s}$. Images were obtained by subtracting an SXR image from another obtained $6.6 \mu$ s later.

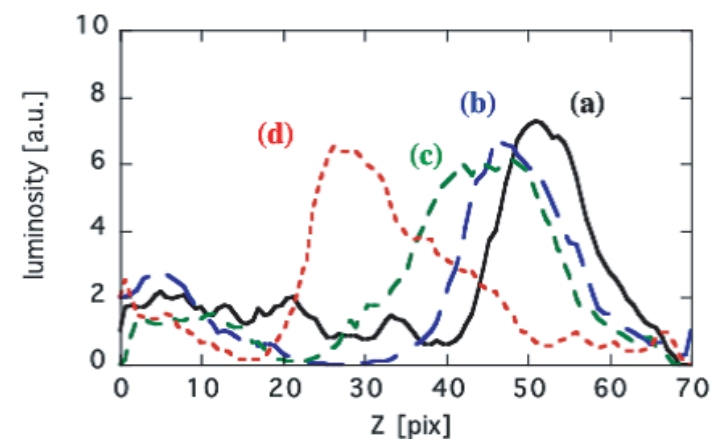

Fig. 6 Time evolution of the vertical (Z) luminosity profiles in SXR images at the center of the viewing circle.

files in the SXR images at the center of the viewing circle. By comparing them, we see clearly that the peak in the luminosity moves toward the vertical direction with time.

Next, we calculated SXR images for some model profiles of the SXR emissivity to clarify the meaning of the configuration in 2-D SXR images such as Fig. 5. In the calculation, the radial position of the magnetic island, i.e., the rational surface, was determined by equilibrium reconstruction from several external diagnostics on RELAX: the plasma current $I_{\mathrm{p}}$, average toroidal field $\left\langle B_{\phi}\right\rangle$, edge toroidal $B_{\phi}(a)$, poloidal field $B_{\theta}(a)$, and internal line-averaged density $n_{\mathrm{e}}$ obtained using an interferometer [13]. The width $W$ of the magnetic island due to the corresponding $m / n$ tearing mode can be estimated from the fluctuation component of the corresponding tearing mode. When neighboring islands are sufficiently broad, they overlapped each other, resulting in a stochastic magnetic field. In this calculation, we assumed a constant radiation power along the magnetic field lines. The phase of a magnetic island $\phi$ was fixed according to experimental data obtained by edge magnetic measurements. The SXR emissivity profiles can be controlled without departing from the results of the equilibrium reconstruction.

Figure 7 shows the simulated SXR image on the MCP calculated assuming the same SXR emissivity inside the island. $\phi$ was determined using the result of mode analysis from edge magnetic measurements, (a) $\phi=-3.08$ for $933 \mu \mathrm{s}$, (b) $\phi=-2.92$ for $940 \mu \mathrm{s}$, (c) $\phi=-2.74$ for $947 \mu \mathrm{s}$, and (d) $\phi=-2.48$ for $953 \mu$ s. Each figure was obtained by subtracting an SXR image from the next consecutive im- 
(a)

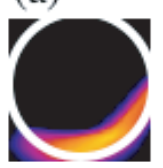

(b)

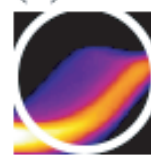

(c)

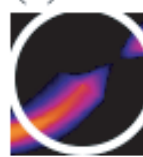

(d)

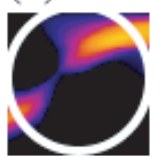

Fig. 7 Simulation of subtracted images: Finite SXR emissivity is assumed only inside the $m=1 / n=4$ magnetic island. The time in each panel is that is the corresponding panel in Fig. 5. The phase change is based on the experimental phase velocity.

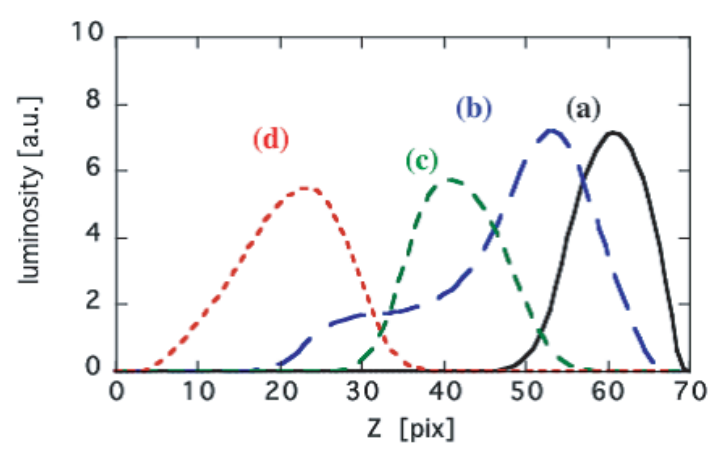

Fig. 8 Evolution of the vertical $(Z)$ luminosity profiles in the simulation of subtracted SXR images.

age. A luminosity distribution resembling the zonal structure in the experimental results was reproduced in the calculated images. Figure 8 shows the evolution of the vertical $(Z)$ luminosity profiles in Fig. 7. A comparison of Figs. 5 and 6 with Figs. 7 and 8 reveals a discrepancy in the peak position; however, the overall characteristics show good agreement. A comparison between the experimental and simulated results suggests that the vertical images in Fig. 5 may indicate rotating helical SXR emissivity. It is clear that we must adjust the island parameters further in order to realize better agreement between the experimental and calculated images. Moreover, we need to obtain more data sets and analyze the transition event between the QSH state and the multi-helicity state.

\section{Summary}

In conclusion, the initial result of successive SXR imaging with a high-speed camera from the vertical port were presented. To identify magnetic islands by referring to the SXR images, we estimated the 2-D image expected from low- $A$ RFP plasmas. We described a preliminary experimental result in which we observed vertical SXR images from the quasi-periodic QSH RFP state. We successfully obtained the time evolution of SXR images from the vertical port. We calculated SXR images for some model profiles of the SXR emissivity to clarify the meaning of the configuration in 2-D SXR images. By comparing the obtained experimental images during a single mode dominant phase with simulated SXR images calculated assuming a rotating helical configuration, we find that the evolution of the experimental SXR image suggests a rotating helical SXR emissivity.

\section{Acknowledgments}

This study was supported by a U.S.-Japan collaboration and a Grant-in-Aid for Scientific Research (No.17360441) from the Ministry of Education, Culture, Sports and Technology, Japan. This study was performed with the support and under the auspices of the National Institute for Fusion Science (NIFS) Collaborative Research Program (NIFS10KOAP024).

[1] S. Ohdachi et al., Plasma Fusion Res. 2, S1016 (2007).

[2] S. Masamune et al., J. Phys. Soc. Jpn. 76, 123501 (2007).

[3] K. Oki et al., J. Phys. Soc. Jpn. 77, 075005 (2008).

[4] R. Ikezoe et al., Plasma Fusion Res. 3, 029 (2008).

[5] T. Onchi et al., Plasma Fusion Res. 2, S1063 (2007).

[6] A. Sanpei et al., Plasma Fusion Res. 2, S1064 (2007).

[7] T. Onchi et al., Rev. Sci. Instrum. 81, 073502 (2010).

[8] A. Sanpei et al., Plasma Fusion Res. 6, 2406096 (2011).

[9] A. Sanpei et al., IEEE Trans. Plasma Sci. 39, 2410 (2011).

[10] G.W. Fraser, Nucl. Instrum. Methods 195, 523 (1982).

[11] Hamamatsu Photonics, MCP assembly technical report, 2007.

[12] I.H. Hutchinson, Principles of Plasma Diagnostics (Cambridge University Press, Cambridge, 1987).

[13] A. Sanpei et al., J. Phys. Soc. Jpn. 78, 013501 (2009). 